УДК 539.374

\title{
Solution of Boundary Value Problems of Plasticity with the Use of Conservation Laws
}

\author{
Sergei I. Senashov* \\ Irina L. Savostyanova ${ }^{\dagger}$ \\ Reshetnev Siberian State University of Science and Technology \\ Krasnoyarsky Rabochy, 31, Krasnoyarsk, 660037 \\ Russia \\ Olga N.Cherepanova ${ }^{\ddagger}$ \\ Institute of Mathematics and Computer Science \\ Siberian Federal University \\ Svobodny, 79, Krasnoyarsk, 660041 \\ Russia
}

Received 20.11.2017, received in revised form 26.01.2018, accepted 20.03.2018

In the paper conservation laws of a special form for systems of first-order differential equations depending on two dependent and independent variables are looked at. It is shown how the conservation laws can be used to solve hyperbolic-type and elliptic-type systems of equations that are come across in the theory of plasticity. Examples of an effective use of the described technique are given. With the use of the conservation laws was found the elastoplastic boundary in a problem of stress-strain state of a plate with free-form holes.

Keywords: conservation laws, elastic-plastic boundary

DOI: 10.17516/1997-1397-2018-11-3-356-363.

\section{Introduction}

Symmetries and conservation laws allow studying of differential equations from different points of view.

Symmetries act on the manifold of solutions of differential equations and that is why allow us to study and find their solutions in two ways. The first way is to find the so-called fixed-points, i.e. such solutions that do not change when some symmetries act. Invariant solutions get found in such a way. This method is realised to the full extent in the papers of L.V.Ovsyannikov [1].

The other approach is frequently declared but is seldom realised. Its main point is that we get symmetries to act on the given solution and we obtain new solutions of the same differential equation system. It was used in the papers [2-5]. There may be an obstruction for realisation of this method which is "poorness" of the admitted group of symmetries or difficulty to interpret the found "multiplied" solutions. We manage to avoid difficulties in interpretation if we act not on the solution itself but on some other objects related to it, for example on characteristics in case of hyperbolic equations. Use of symmetries when solving boundary value problems for

\footnotetext{
*sen@sibsau.ru

†savostyanova@sibsau.ru

${ }^{\ddagger}$ cheronik@mail.ru

(c) Siberian Federal University. All rights reserved
} 
differential equation systems encounters significant difficulties, though attempts of such a kind have been undertaken, this is evidently explained by local property of symmetries. But on the other hand the technique of using symmetries allows finding and describing fairly wide classes of solutions, and at that fairly effectively.

Conservation laws building technique is in actual fact dual to symmetries building technique and the methods to find them are close to the methods to find symmetries. But the results significantly differ: conservation laws allow solving boundary value problems, and at that fairly effectively. This is illustrated in a series of papers on solving of boundary value problems for plasticity equations and other continuum mechanics equations [6-10].

\section{Fundamental definitions}

For simplicity we restrict ourselves to the case of differential equations depending on two independent $\mathrm{x}, \mathrm{y}$ and two dependent variables $u, v$. This case is simpler for understanding; best investigated and is frequently come across in mechanics problems. Let us look at the system of the two differential equations

$$
F_{1}=F_{1}\left(x, y \cdot u, v \cdot u_{x}, u_{y}, v_{x}, v_{y}\right)=0, \quad F_{2}=F_{2}\left(x, y \cdot u, v \cdot u_{x}, u_{y}, v_{x}, v_{y}\right)=0,
$$

where the index below stands for a derivative with respect to the corresponding argument.

Definition 1. As the conservation law for system of equations (1) we will call the expression in the form of

$$
D_{x} A+D_{y} B=\Omega_{1}\left(F_{1}\right)+\Omega_{2}\left(F_{2}\right),
$$

where $\Omega_{i} \mathrm{i}=1,2$ some linear differential operators that at the same time are not trivial, $D_{x}, D_{y}$ are operators of full differentiation for the corresponding variables $D_{x}=\partial_{x}+u_{x} \partial_{u}+v_{x} \partial_{v}+\ldots$, $D_{y}=\partial_{y}+u_{y} \partial_{u}+v_{y} \partial_{v}+\ldots$.

Remark. This quite a commonplace definition is reasonably suitable for the presentation of our results. More general approach can be learned in [11].

Definition 2. Vector $(A, B)$ is called conserved current.

In this paper functions and are supposed to be dependent on $x, y, u, v$ only. We can assume that and also depend on the derivatives but such conservation laws for system (2) are difficult to interpret and that is why they will not be considered here.

From (1) according to Riemann formula it follows

$$
\oint_{\mathbb{C}} A \mathrm{~d} y-B \mathrm{~d} x=0
$$

where $\mathbb{C}$ is closed contour in which Functions and have no singularities for simplicity. Let us show how conservation laws (2) can be used for different types of differential equations.

\section{Use of conservation laws to solve hyperbolic systems of differential equations}

Assume (1) is a system of hyperbolic differential equations. Its characteristics are written as

$$
\Gamma_{1}: \frac{\mathrm{d} y}{\mathrm{~d} x}=\alpha(u, v, x, y), \quad \Gamma_{2}: \frac{\mathrm{d} y}{\mathrm{~d} x}=\beta(u, v, x, y) .
$$


These characteristics proceed from the endpoints of the segment of curve $L x_{1} \leqslant x \leqslant x_{2}$ with conditions established on it

$$
\left.u\right|_{L}=u_{0},\left.v\right|_{L}=v_{0} .
$$

Assume $\left(x_{p}, y_{p}\right)$ is the point of intersection of the characteristics (see Fig. 1).

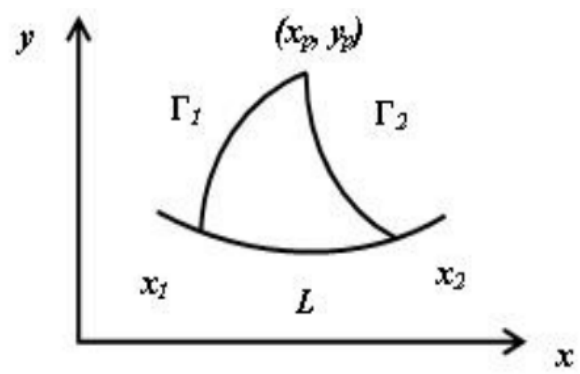

Fig. 1

Let us find this point. By virtue of (3) we have

We have

$$
\int_{L} A \mathrm{~d} y-B \mathrm{~d} x+\int_{\Gamma_{1}} A \mathrm{~d} y-B \mathrm{~d} x+\int_{\Gamma_{2}} A \mathrm{~d} y-B \mathrm{~d} x=0 .
$$

$$
\begin{aligned}
& \int_{\Gamma_{1}} A \mathrm{~d} y-B \mathrm{~d} x=\int_{\Gamma_{1}}(A-\alpha B) \mathrm{d} y=\left.(A-\alpha B) y\right|_{y_{p}} ^{y_{1}}-\int_{\Gamma_{1}} y \mathrm{~d}(A-\alpha B) \\
& \int_{\Gamma_{2}} A \mathrm{~d} y-B \mathrm{~d} x=\int_{\Gamma_{2}}(A-\beta B) \mathrm{d} y=\left.(A-\beta B) y\right|_{y_{p}} ^{y_{1}}-\int_{\Gamma_{2}} y \mathrm{~d}(A-\beta B) .
\end{aligned}
$$

Let us assume that and is the solution of equation (2) with the following boundary conditions $\left.(A-\alpha B)\right|_{\Gamma_{1}}=$ const, $\left.(A-\beta B)\right|_{\Gamma_{2}}=$ const.

In this case from (5) we can obtain coordinate $y_{p}$. In the same way coordinate $x_{p}$ shall be obtained. In this way we manage to build the characteristics of System of equations (1), and therefore to solve the set problem. Moreover for many of mechanics problems building of the characteristics often give more information to the researcher than finding of an explicit solution. The described method is effective, with the use of which were solved Cauchy and Riemann problems for perfect plasticity equations, and also some problems for other equations of mechanics [12].

\section{Use of conservation laws to solve elliptic of differential equation systems}

Assume system (1) is of an elliptic type. We will be finding the solution of equation (2) that has a singularity in point $\left(x_{0}, y_{0}\right)$. Assume that on closed curve $L$ the functions $\left.u\right|_{L}=u_{0},\left.v\right|_{L}=v_{0}$ are defined.

Let us encircle point $\left(x_{0}, y_{0}\right)$ with a circumference with a radius $\varepsilon:\left(x-x_{0}\right)^{2}+\left(y-y_{0}\right)^{2}=\varepsilon^{2}$.

Let us connect it as shown on Fig. 2, with a cut with contour $L$.

We have

$$
\int_{L} A \mathrm{~d} y-B \mathrm{~d} x+\int_{\Gamma_{1}} A \mathrm{~d} y-B \mathrm{~d} x+\int_{\Gamma_{2}} A \mathrm{~d} y-B \mathrm{~d} x+\int_{\varepsilon} A \mathrm{~d} y-B \mathrm{~d} x=0 .
$$




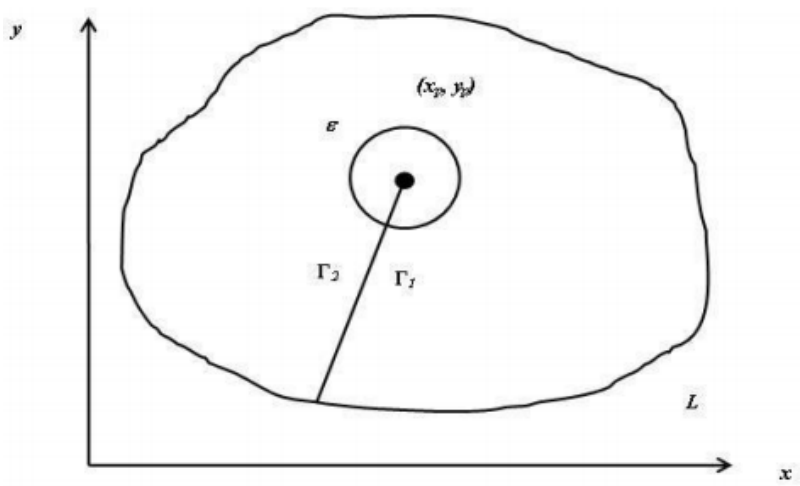

Fig. 2

From (6) we obtain

$$
\int_{L} A \mathrm{~d} y-B \mathrm{~d} x=-\int_{\varepsilon} A \mathrm{~d} y-B \mathrm{~d} x
$$

Let us set $\varepsilon \rightarrow 0$. We obtain a relation relating $u\left(x_{0}, y_{0}\right), v\left(x_{0}, y_{0}\right)$ and $\int_{L} A \mathrm{~d} y-B \mathrm{~d} x$. Having chosen another conserved current with the same conditions we get another relation relating $u\left(x_{0}, y_{0}\right), v\left(x_{0}, y_{0}\right)$ and $\int_{L} A \mathrm{~d} y-B \mathrm{~d} x$. These two relations allow finding values $u\left(x_{0}, y_{0}\right), v\left(x_{0}, y_{0}\right)$ and because point $\left(x_{0}, y_{0}\right)$ is arbitrary, then therefore the solution for the set problem has been built. This method is realised in papers [8-10]. It allowed calculating of the boundary between plastic strain range and elastic strain range for rolled section rods being twisted. The results of the calculations are given in the Fig. 3.

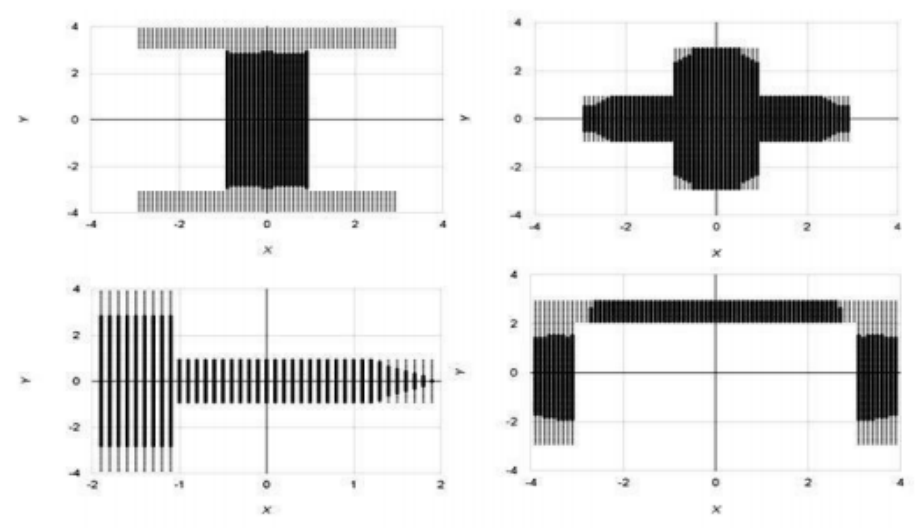

Fig. 3

\section{Solution of a plane elastic-plastic problem}

Within the plastic strain range in a bidimensional case with a general yield criterion the equations will be written as

$$
\frac{\partial \sigma_{x}}{\partial x}+\frac{\partial \tau}{\partial y}=0, \quad \frac{\partial \tau}{\partial x}+\frac{\partial \sigma_{y}}{\partial y}=0
$$




$$
\left(\sigma_{x}, \sigma_{y}, \tau\right)=0
$$

Within the elastic strain range relations (7) and compatibility condition of deformations are performed that is written as

$$
\begin{aligned}
& \frac{\partial \sigma_{x}}{\partial x}+\frac{\partial \tau}{\partial y}=0, \quad \frac{\partial \tau}{\partial x}+\frac{\partial \sigma_{y}}{\partial y}=0 \\
& \frac{\partial^{2}\left(\sigma_{x}+\sigma_{y}\right)}{\partial x^{2}}+\frac{\partial^{2}\left(\sigma_{x}+\sigma_{y}\right)}{\partial y^{2}}=0 .
\end{aligned}
$$

\section{Problem setting}

Let us consider a finite rectangular plate with dimensions of $a \times b$ with holes of an arbitrary shape limited by contours $\Gamma_{1}, \Gamma_{2}, \ldots, \Gamma_{\mathrm{m}}$. Let us assume that the plate is under different stresses in $x, y$ directions, and the contour lines of the holes are under the action of the set stresses.

On the external boundaries of $\Gamma_{0}$ we get the following conditions

$$
\left.\sigma_{x}\right|_{x=a}=\left.\sigma_{x}\right|_{x=0}=q_{1},\left.\quad \sigma_{y}\right|_{y=b}=\left.\sigma_{y}\right|_{y=0}=q_{2} .
$$

Other stress components on these boundaries are equal to zero.

On the boundary of contours $\Gamma_{1}, \Gamma_{2}, \ldots, \Gamma_{\mathrm{m}}$ the condition is fulfilled.

$$
\sigma_{x} n^{i}+\left.\tau m^{i}\right|_{\Gamma^{i}}=p_{1}^{i}, \quad \tau n^{i}+\left.\sigma_{y} m^{i}\right|_{\Gamma^{i}}=p_{2}^{i}
$$

where $q_{i}, p_{j}^{i}$ are constants, $\left(n^{i}, m^{i}\right)$ is vector components of the normal to contour $\Gamma_{\mathrm{i}}$.

We assume that stresses $\sigma_{x}, \sigma_{y}, \tau$ on contours $\Gamma_{1}, \Gamma_{2}, \ldots, \Gamma_{\mathrm{m}}$ are such that plasticity condition (8) is fulfilled.

From equation (9) we obtain

$$
\sigma_{x}+\sigma_{y}=F(x, y)
$$

where $F(x, y)$ some solution of Laplace equation (9). From (10) and (11) it follows that in order to find $F(x, y)$ we need to solve Laplace equation with the following boundary conditions

$$
\left.F\right|_{x=a}=\left.F\right|_{x=0}=q_{1},\left.\quad F\right|_{y=b}=\left.F\right|_{y=0}=q_{2},\left.\quad F\right|_{\Gamma^{i}}=d^{i} .
$$

Value $d^{i}$ shall be determined from the solution of the three equations (11) and (8).

Let us consider the solution of problem (12), (13) as known because this is a classical problem for Laplace equation. In this case in order to find $\sigma_{x}, \tau$ we will get the system of equations

$$
\Phi_{1}=\frac{\partial \sigma_{x}}{\partial x}+\frac{\partial \tau}{\partial y}=0, \quad \Phi_{2}=\frac{\partial \tau}{\partial x}-\frac{\partial \sigma_{x}}{\partial y}+\frac{\partial F}{\partial y}=0
$$

For system (14) let us write conservation laws in the form of (2)

$$
D_{x} A+D_{y} B=\Omega_{1}\left(\Phi_{1}\right)+\Omega_{2}\left(\Phi_{2}\right) .
$$

Let us find conserved current in the form of:

$$
\begin{aligned}
& A=\alpha_{1}(x, y) \sigma_{x}+\beta_{1}(x, y) \tau+\gamma_{1}(x, y), \\
& B=\alpha_{2}(x, y) \sigma_{x}+\beta_{2}(x, y) \tau+\gamma_{2}(x, y) .
\end{aligned}
$$


As a result we have

$$
\frac{\partial \alpha_{1}}{\partial x}-\frac{\partial \beta_{1}}{\partial y}=0, \quad \frac{\partial \beta_{1}}{\partial x}+\frac{\partial \alpha_{1}}{\partial y}=0, \quad \frac{\partial \gamma_{1}}{\partial x}+\frac{\partial \gamma_{2}}{\partial y}=-\beta_{1} \frac{\partial F}{\partial y} .
$$

Finally conserved current shall be written as

$$
\begin{aligned}
& A=\alpha_{1}(x, y) \sigma_{x}+\beta_{1}(x, y) \tau+\gamma_{1}(x, y), \\
& B=-\beta_{1}(x, y) \sigma_{x}+\alpha_{1}(x, y) \tau+\gamma_{2}(x, y),
\end{aligned}
$$

coefficients of which are related by relations (16).

To simplify further calculations let us suppose that we have only one hole limited by contour $\Gamma_{1}$, (see Fig. 4) according to Green formula we get

$$
\oint_{\Gamma_{0}} A \mathrm{~d} y-B \mathrm{~d} x+\oint_{\Gamma_{1}} A \mathrm{~d} y-B \mathrm{~d} x=0 .
$$

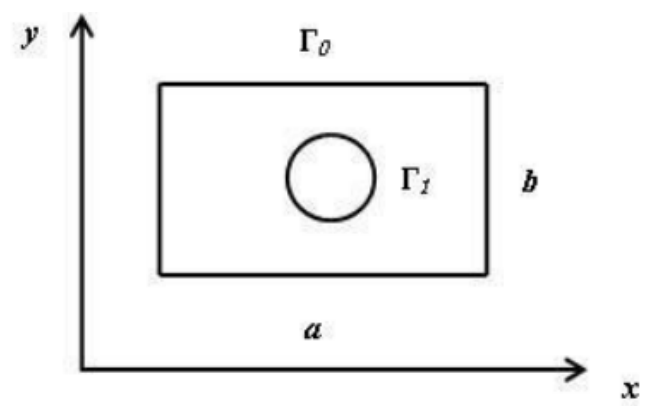

Fig. 4

Let us look at the two singular solutions of equations (16)

$$
\begin{aligned}
& \alpha_{1}^{1}=\frac{x-x_{0}}{\left(x-x_{0}\right)^{2}+\left(y-y_{0}\right)^{2}}, \quad \beta_{1}^{1}=-\frac{y-y_{0}}{\left(x-x_{0}\right)^{2}+\left(y-y_{0}\right)^{2}}, \\
& \gamma_{2}^{1}=-\int^{\frac{\partial F}{\partial y}} \omega_{2} d y, \quad \gamma_{1}^{1}=0 . \\
& \alpha_{1}^{2}=\frac{y-y_{0}}{\left(x-x_{0}\right)^{2}+\left(y-y_{0}\right)^{2}}, \quad \beta_{1}^{2}=\frac{x-x_{0}}{\left(x-x_{0}\right)^{2}+\left(y-y_{0}\right)^{2}}, \\
& \gamma_{2}^{2}=-\int^{\frac{\partial F F}{\partial y}} \omega_{2} \mathrm{~d} y, \quad \gamma_{1}^{2}=0 .
\end{aligned}
$$

From (19) and (20) according to the above shown formulas we finally obtain

$$
\begin{aligned}
2 \pi \sigma_{x}\left(x_{0}, y_{0}\right)= & -\int_{\Gamma_{0}}\left(\frac{y-y_{0}}{\left(x-x_{0}\right)^{2}+\left(y-y_{0}\right)^{2}} \sigma_{x}-\frac{x-x_{0}}{\left(x-x_{0}\right)^{2}+\left(y-y_{0}\right)^{2}} \tau\right) \mathrm{d} y- \\
& -\left(\frac{y-y_{0}}{\left(x-x_{0}\right)^{2}+\left(y-y_{0}\right)^{2}} \sigma_{x}+\frac{x-x_{0}}{\left(x-x_{0}\right)^{2}+\left(y-y_{0}\right)^{2}} \tau+\gamma_{1}^{1}\right) \mathrm{d} x-\int_{\Gamma_{1}} \gamma_{1}^{1} d y, \\
2 \pi \tau\left(x_{0}, y_{0}\right)= & -\int_{\Gamma_{0}}\left(\frac{x-x_{0}}{\left(x-x_{0}\right)^{2}+\left(y-y_{0}\right)^{2}} \sigma_{x}+\frac{y-y_{0}}{\left(x-x_{0}\right)^{2}+\left(y-y_{0}\right)^{2}} \tau\right) \mathrm{d} y- \\
& -\left(-\frac{x-x_{0}}{\left(x-x_{0}\right)^{2}+\left(y-y_{0}\right)^{2}} \sigma_{x}+\frac{y-y_{0}}{\left(x-x_{0}\right)^{2}+\left(y-y_{0}\right)^{2}} \tau+\gamma_{1}^{2}\right) \mathrm{d} x-\int_{\Gamma_{1}} \gamma_{1}^{2} d y,
\end{aligned}
$$




$$
\sigma_{y}\left(x_{0}, y_{0}\right)=F\left(x_{0}, y_{0}\right)-\sigma_{x}\left(x_{0}, y_{0}\right)
$$

We insert the obtained stresses into yield condition (8). In those points where $f\left(\sigma_{x}, \sigma_{y}, \tau\right)<0$, it will be elastic range, and other points of the plate belong to plastic range.

\section{Conclusion}

The results of the use of conservation laws to solve boundary value problems have shown that this method is effective for the problems of the theory of plasticity and elastic plasticity.

\section{References}

[1] L.V.Ovsyannikov, Group Analysis of Differential Equations, NewYork, Academic Press, 1982.

[2] S.I.Senashov, A.N.Yakhno, Reproduction of solutions for bi-dimensional ideal plasticity, Journal of Non-Linear Mechanics, 42(2007), 500-503.

[3] S.I.Senashov, A.N.Yakhno, Deformation of characteristic curves of the plane ideal plasticity equations by point symmetries, Nonlinear analysis , 71(2009), 1274-1284.

[4] S.I.Senashov, O.N.Cherepanova, New classes of solution of minimal surfaces, J. Siberian Federal Univ., Math. and Physics., 3(2010), no. 2, 248-255 (in Russian).

[5] S.I.Senashov, Conservation Laws. Hodograph Transformation and Boundary Value Problems of Plane Plasticity, SIGMA, 8(2012), 071.

[6] S.I.Senashov, A.N.Yakhno, Some symmetry group aspects of a perfect plane plasticity system, J. Phys. A: Math. Theor, 46(2013), 355202.

[7] S.I.Senashov, A.N.Yakhno, Conservation Laws of Three-Dimensional Perfect Plasticity Equations under von Mises Yield Criterion, Abstract and Applied Analysis, 2013(2013), $702-732$.

[8] S.I.Senashov, A.V.Kondrin, O.N.Cherepanova, On Elastoplastic Torsion of a Rod with Multiply Connected Cross-Section, J. Siberian Federal Univ., Math. and Physics., 7(2015), no. 1, $343-351$.

[9] S.I.Senashov, O.N.Cherepanova, A.V.Kondrin, Elasto-plastic Bending of Beam, J. Siberian Federal Univ., Math. and Physics., 7(2014), no. 2, 218-223.

[10] S.I.Senashov, E.V.Filyushina, O.V.Gomonova, Construction of elasto-plastic boundaries using conservation laws, Vestnik SibGAU, 16(2015), no. 2, 343-359.

[11] S.I.Senashov, A.M.Vinogradov, Symmetries and Conservation Laws of 2-Dimensional Ideal Plasticity, Proc. of Edinb. Math. Soc, 31(1988), 415-439.

[12] S.I.Senashov, P.P.Kiriakov, A.N.Yakhno, Application of symmetries and conservation laws of solution of Differential Equation, Novosibirsk, Nauka, 2001 (in Russian). 


\section{Решение краевых задач пластичности с использованием законов сохранения}

Сергей И. Сенашов

Ирина Л. Савостьянова

Сибирский государственный университет науки и технологий им. М. Ф. Решетнева Красноярский рабочий, 31, Красноярск, 660037

Россия

Ольга Н. Черепанова

Институт математики и фундаментальной информатики Сибирский федеральный университет

Свободный, 79, Красноярск, 660041

Россия

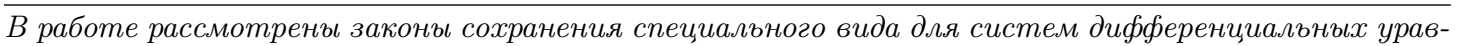
нений первого порядка, зависящие от двух зависимых и независимых переменных. Показано как законы сохранения могут быть использованы для решения систем уравнений гиперболического и эллиптического типов, которые встречаются в теории пластичности. Приведены примеры эффективного применения описанной методики. С помощью законов сохранения найдена упругопластическая граница в задаче о напряженно дебормированном состоянии пластины с отверстиями произвольной формы.

Ключевые слова: законы сохранения, упругопластическая граница. 